\title{
Competências que graduandos em fisioterapia precisam desenvolver na visão de fisioterapeutas da urgência e emergência
}

\author{
Competences that physical therapy undergraduate students need to develop in the view of \\ physiotherapists who work at emergency department
}
Competencias que los estudiantes universitarios de fisioterapia necesitan desarrollar en la vista de los fisioterapeutas en urgencias y emergencias

Kéven Lorena de Paula Gonçalves ${ }^{1 *}$, Renato da Costa Teixeira².

\begin{abstract}
RESUMO
Objetivo: Descrever as competências profissionais para atuação na Urgência e Emergência (UE) que alunos do curso de Fisioterapia devem construir, de acordo com a experiência de fisioterapeutas da área. Métodos: Trata-se de um estudo transversal, descritivo, de abordagem qualiquantitativa. Como método para coleta de dados foram realizadas entrevistas semiestruturadas, cuja análise de conteúdo foi realizada no software IRaMuTeQ (versão 0.7). A amostra foi composta por 11 fisioterapeutas que atuam em um hospital de referência no atendimento às urgências traumáticas. Resultados: Os resultados obtidos permitiram a elaboração de um rol de conhecimentos, habilidades e atitudes mínimos, no contexto de uma formação generalista, que os alunos do curso de Graduação em Fisioterapia precisam desenvolver para atuação profissional na Urgência e Emergência, como manejo da ventilação mecânica, manobras de reanimação cardiopulmonar, interpretação de exames, avaliação fisioterapêutica, trabalho em equipe, comunicação efetiva, etc. Conclusão: Concluise que os resultados deste estudo podem contribuir para o planejamento de estratégias de ensino-aprendizagem dos cursos de Fisioterapia voltadas para o campo da UE no âmbito de uma formação generalista.
\end{abstract}

Palavras-chave: Curso de fisioterapia, Competência profissional, Atendimento de urgência.

\begin{abstract}
Objective: To describe the professional competences to work in the Emergency Department (ED) that Physiotherapy undergraduates need to build, in the perspective of physiotherapists in the ED area. Methods: This is a cross-sectional, descriptive study with a qualitative approach. As a method for data collection, semi-structured interviews were conducted, whose content analysis was performed in the software IRaMuTeQ (version 0.7). The sample consisted of 11 physical therapists who work at a reference hospital in traumatic emergencies. Results: The results obtained allowed the elaboration of a list of minimum knowledge, skills and attitudes, in the context of a generalist education, that the undergraduate Physiotherapy students need to develop for professional performance in Emergency Department, such as management of mechanical ventilation, cardiopulmonary resuscitation maneuvers, interpretation of tests, physical therapy assessment, teamwork, effective communication, etc. Conclusion: It is concluded that the results of this study can contribute to the planning of teaching-learning strategies for Physiotherapy courses aimed at the ED field within the scope of generalist training.
\end{abstract}

Keywords: Physical therapy specialty, Professional competence, Emergency care.

\section{RESUMEN}

Objetivo: Describir las competencias profesionales para trabajar en la Urgencia e Emergencia (UE) que los estudiantes de Fisioterapia necesitan desarrollar desde la perspectiva de los fisioterapeutas de esta área. Métodos: Se trata de un estudio descriptivo transversal con abordaje cualitativo. Como método de recolección de datos se realizaron entrevistas semiestructuradas, cuyo análisis de contenido se realizó en el software IRaMuTeQ (versión 0.7). La muestra estuvo conformada por 11 fisioterapeutas que laboran en un hospital de referencia en emergencias traumáticas. Resultados: Los resultados obtenidos permitieron la elaboración de un listado de conocimientos, habilidades y actitudes mínimos, en el contexto de una educación generalista, que los estudiantes de Fisioterapia necesitan desarrollar para el desempeño profesional en Urgencias y Emergencias, tales como manejo de ventilación mecánica, maniobras de reanimación cardiopulmonar, interpretación de pruebas, valoración de fisioterapia, trabajo en equipo, comunicación efectiva, etc. Conclusión: Se concluye que los resultados de este estudio pueden contribuir a la planificación de estrategias de enseñanza-aprendizaje para los cursos de Fisioterapia dirigidos al ámbito de la UE, dentro de una formación generalista.

Palabras clave: Especialidad de Fisioterapia, Competencia profesional, Atención ambulatoria.

${ }^{1}$ Faculdade Cosmopolita, Belém - PA. *E-mail: keven.lorena@hotmail.com

2 Universidade do Estado do Pará (UEPA), Belém - PA. 


\section{INTRODUÇÃO}

Os serviços de Urgência e Emergência (UE) diariamente escancaram um sério problema de Saúde Pública, com cenários de superlotação, sofrimento, longa espera e, até mesmo, caos. A alta demanda por atendimentos de urgência ocorre em paralelo e como consequência da baixa resolutividade da Atenção Básica e da transição epidemiológica brasileira em que se observa uma "tripla carga de doença", com ressurgimento/persistência de casos de doenças infectocontagiosas, emergência das doenças crônicodegenerativas e aumento significativo dos acidentes trânsito e violência urbana (PINES J, et al., 2011; MORAES EN, 2012; MORAES JC, 2013; FRAGA GP, 2016).

Desta forma, no cenário caótico das urgências, onde há sobrecarga também dos profissionais que tradicionalmente atuam nesses serviços (médicos, enfermeiros e técnicos de enfermagem), inserção de outros profissionais, que podem contribuir em seu escopo de atuação para a resolução das demandas apresentadas, têm crescido nos últimos anos (JESUDASON C, et al., 2012).

Uma vez que novos papéis profissionais vêm surgindo nesses serviços, abre-se espaço para a atuação do fisioterapeuta com o objetivo de prestar assistência à saúde, melhorar o serviço prestado e sua resolutividade e, consequentemente, aumentar a satisfação dos usuários. Nesse contexto, ressalta-se a importância da seleção de profissionais capacitados, e o processo de formação, então, se destaca como forma de capacitação para atuação na UE, e como meio para a busca de uma melhor definição do papel do fisioterapeuta e expansão deste campo de atuação, com o desenvolvimento de pesquisas (SALT E, 2016; SUTTON M, et al., 2015; TAYLOR N, et al., 2011; FLEMING-MCDONNELL D, et al., 2010; JESUDASON C, et al., 2012).

Por isso, os estágios supervisionados são componentes curriculares essenciais para a construção de competências, pois permite a mobilização de conhecimentos, habilidades e atitudes que servirão como base para a resolução de problemas do cotidiano profissional em uma situação real. Pesquisas na área de Fisioterapia na UE podem fortalecer e concretizar a atuação do profissional nesse campo e garantir dados que possam subsidiar uma prática segura e baseada em evidências científicas (MARINHO-ARAÚJO CM e ALMEIDA LS, 2016; FRUTH SJ e WILEY S, 2016).

O objetivo desta pesquisa foi descrever quais são as competências que devem ser desenvolvidas pelos graduandos em Fisioterapia, na perspectiva de uma formação generalista, a fim de prepará-los para atuar na UE, na visão de fisioterapeutas que trabalham nesse setor.

\section{MÉTODOS}

Trata-se de um estudo transversal, descritivo, de abordagem qualiquantitativa, desenvolvido em um hospital de referência no atendimento de urgências e emergências de origem traumática, situado na Região Norte do Brasil. A pesquisa recebeu aprovação (parecer de número 2.161.317) do Comitê de Ética em Pesquisa da Universidade do Estado do Pará (UEPA) e obedeceu às normas regulamentadoras de pesquisas envolvendo seres humanos.

Os participantes da pesquisa foram os fisioterapeutas que exercem atividades assistenciais aos pacientes do hospital e recebem os alunos de Fisioterapia em estágio supervisionado de Instituições de Ensino Superior (IES) públicas e/ou privadas. Foram incluídos os fisioterapeutas que atuam no setor de pronto-atendimento, há, pelo menos, 1 ano, e trabalham em setores que recebem alunos de Fisioterapia. Foram excluídos os fisioterapeutas que atuam na docência, pois acredita-se que seus conhecimentos prévios e experiências como professores poderiam influenciar em suas respostas mais do que a prática na UE em si.

Foi realizada uma entrevista individual semiestruturada com os fisioterapeutas que atenderam aos critérios de inclusão. O conteúdo da entrevista foi áudio-gravado e transcrito na íntegra. Considerando os aspectos éticos, os entrevistados foram identificados como "Fisio 1", "Fisio 2", "Fisio 3" e assim sucessivamente, de acordo com a ordem das entrevistas.

As transcrições foram submetidas à análise de conteúdo com o emprego da técnica de Classificação Hierárquica Descendente (CHD) no software Interface de R pour les Analyses Multidimensionnelles de Textes et de Questionnaires (IRaMuTeQ, versão 0.7), a partir do corpus colorido criado pelo software, o que possibilitou a distribuição das categorias de análise de acordo com o conteúdo de cada entrevista. 
O perfil dos fisioterapeutas entrevistados foi gerado com o uso do software Microsoft ${ }^{\circledR}$ Office Excel ${ }^{\circledR}(2010)$, por meio dos cálculos de frequências absolutas e relativas, médias e desvio padrão.

\section{RESULTADOS E DISCUSSÃO}

\section{Perfil dos fisioterapeutas}

Onze fisioterapeutas compuseram a amostra deste estudo, o que corresponde à totalidade dos profissionais que preencheram os critérios de inclusão e exclusão (Figura 1). Foi descrito um resumo do perfil dos fisioterapeutas entrevistados (Tabela 1).

Figura 1 - Amostra da pesquisa segundo os critérios de inclusão e exclusão.

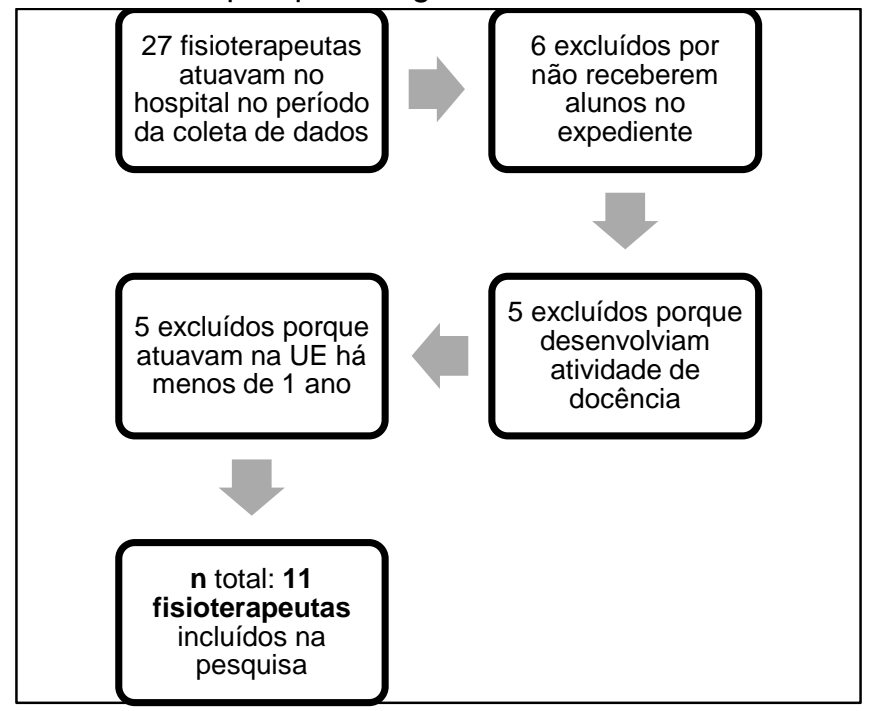

Fonte: Gonçalves KLP e Teixeira RC, 2021.

Tabela 1 - Perfil dos fisioterapeutas entrevistados.

\begin{tabular}{|c|c|}
\hline VARIÁVEL & PERFIL \\
\hline Idade & Média: 29,9 anos $( \pm 3,88)$ \\
\hline Sexo & $\begin{array}{l}\text { Feminino: } n=6 ; 54,5 \% \\
\text { Masculino: } n=5 ; 45,5 \%\end{array}$ \\
\hline Tempo de formação & Média: 7,45 anos $( \pm 4,13)$ \\
\hline Cursos pós-formação ${ }^{1}$ & $\begin{array}{l}\text { Especialização em Fisioterapia em UTI: } n=7 ; 38,89 \% \\
\text { Especialização em Fisioterapia Hospitalar: } n=5 ; 27,78 \% \\
\text { ATLS/BLS }: n=2 ; 11,11 \% \\
\text { Outros }^{3}: n=2 ; 11,11 \% \\
\text { Fisioterapia Respiratória: } n=1 ; 5,56 \%\end{array}$ \\
\hline Especialidade referida ${ }^{4,5}$ & $\begin{array}{l}\text { Fisioterapia em UTI: } n=7 ; 58,33 \% \\
\text { Fisioterapia Respiratória: } n=5 ; 41,67 \%\end{array}$ \\
\hline Título de especialista pelo COFFITO & $\begin{array}{l}\text { Não: } n=9 ; 81,82 \% \\
\text { Sim: } n=2 ; 18,18 \%\end{array}$ \\
\hline Tempo de atuação no HMUE & Média: 4,18 anos $( \pm 2,89)$ \\
\hline Atuação em outros hospitais & $\begin{array}{l}\text { Sim: } n=7 ; 63,64 \% \\
\text { Não: } n=4 ; 36,36 \%\end{array}$ \\
\hline
\end{tabular}

Legenda: 1 Foram relatados 18 cursos pelos fisioterapeutas entrevistados; ${ }^{2}$ Advanced Trauma Life Support/Basic Life Support; ${ }^{3}$ A categoria "Outros" contém os cursos mencionados apenas uma vez e que não têm aplicabilidade direta no âmbito hospitalar, segundo o julgamento da pesquisadora, e foram: cursos de Fisioterapia Aquática, Geriatria e Gerontologia, Reabilitação Vestibular e Perícia Judicial; ${ }^{4}$ Foi considerada neste tópico a especialidade com a qual o fisioterapeuta se apresenta (no currículo, em entrevistas etc.), independentemente dos cursos de especialização ou títulos de especialização profissional; 5 Um fisioterapeuta entrevistado $(n=1)$ referiu duas diferentes especialidades neste tópico.

Fonte: Gonçalves KLP e Teixeira RC, 2021. 


\section{Análise do corpus colorido}

Foram definidas 3 categorias distintas para análise dos resultados: 1 . O cotidiano na UE e a presença de alunos; 2. Conhecimentos e habilidades para atuação na UE; e 3. Atitudes necessárias para atuar na UE e a importância do trabalho em equipe. A primeira categoria de análise não demonstrou relevância para o objetivo desta pesquisa, por isso seu conteúdo foi desconsiderado. As categorias 2 e 3 elencaram os conhecimentos, habilidades e atitudes para a atuação do aluno/futuro profissional de Fisioterapia na UE (Quadro 1).

Quadro 1 - Resumo dos conhecimentos, habilidades e atitudes que precisam ser desenvolvidas pelos alunos de fisioterapia durante estágio supervisionado em UE, na visão dos fisioterapeutas entrevistados.

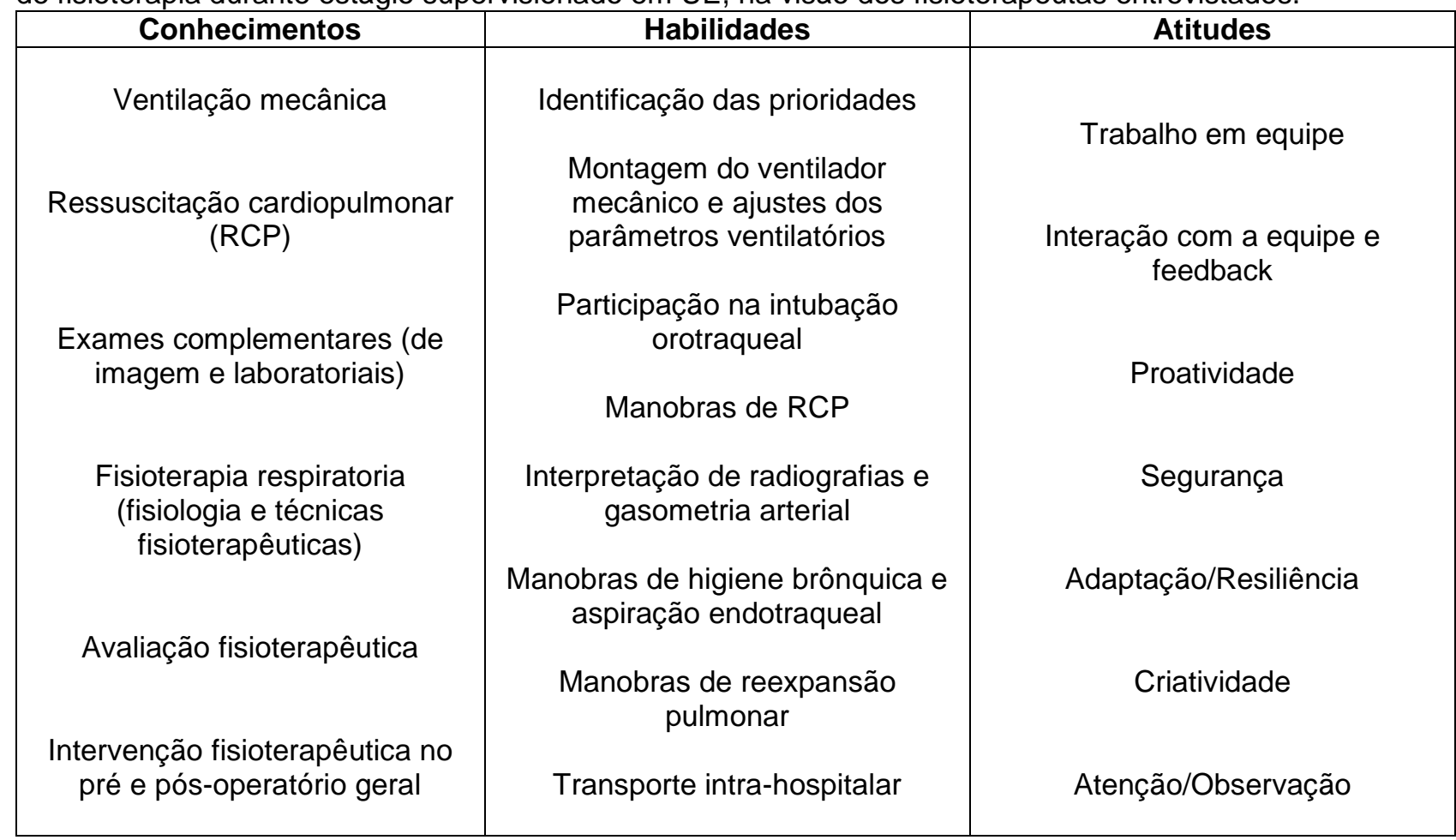

Fonte: Gonçalves KLP e Teixeira RC, 2021.

Elencar competências mínimas para atuação na UE parece ser uma estratégia viável para o direcionamento dos processos de formação que se orientam pela realidade do mercado de trabalho. Entretanto, ressalta-se que as competências elementares podem variar de acordo com o perfil de cada serviço (LEFMANN S e CRANE J, 2016). O hospital onde a pesquisa foi realizada é um serviço de referência em atendimento a pacientes politraumatizados e grandes queimados, ou seja, pacientes mais graves. Por isso, é esperado um perfil de pacientes críticos. Tal fato interfere diretamente nos tipos de condutas fisioterapêuticas empregadas na UE e, certamente, influenciaram nas respostas à entrevista deste estudo.

Kilner E e Shepard L (2010) traçaram o perfil de fisioterapeutas que atuavam na UE na Austrália e encontraram alguns resultados semelhantes ao observado neste estudo: predominância do sexo feminino $(71 \%, n=20 / 28)$, idade entre 30 e 39 anos $(57 \%, n=16 / 28)$, tempo de formação entre 5 e 10 anos $(57 \%, n=$ $16 / 28)$ e a maior parte trabalhava há mais de dois anos na UE $(36 \%, n=10 / 28)$. A maior diferença em comparação foi a especialização na área de trauma-ortopedia (39\%, $n=11 / 28)$ e os cursos pós-formação mais citados foram de radiologia e imobilizações com gesso $(61 \%, n=17 / 28)$. Acredita-se que esta diferença de perfil profissional esteja relacionada às características dos serviços e da forma de atuação da fisioterapia hospitalar no Brasil (mais voltada para atenção aos pacientes críticos).

Por isso, o perfil de formação apresentado pelos fisioterapeutas era esperado, considerando que no contexto em análise as especialidades que provavelmente atuarão mais frequentemente serão: Fisioterapia Cardiovascular, Fisioterapia Respiratória e Fisioterapia em Terapia Intensiva. Não foram encontrados estudos brasileiros que descrevessem a atuação do fisioterapeuta mais voltada para afecções traumato-ortopédicas, 
ao contrário do que se observa no contexto internacional. Apesar de ser um hospital referência em trauma, os pacientes assistidos pelo fisioterapeuta na UE são pacientes críticos, com perfil de Unidade de Terapia Intensiva (UTI), o que requer o perfil profissional mencionado.

No Brasil, observou-se uma grande expansão da Fisioterapia Respiratória, iniciado entre as décadas de 1940 e 1950, período da crise da poliomielite. A partir de então, o fisioterapeuta tem sido reconhecido como membro importante da equipe multiprofissional que presta assistência aos pacientes críticos, o que foi fundamental para a sua inserção definitiva no âmbito hospitalar, na UTI e, mais recentemente (e ainda em andamento), na UE (ALVES AN, 2012).

\section{Conhecimentos e habilidades para atuação na UE}

Devido à urgência do atendimento ao paciente e sua gravidade clínica, é necessário que o fisioterapeuta direcione sua conduta para o que seria o essencial naquele momento, enquanto outras condutas podem ser empregadas quando o paciente estiver estabilizado, na UTI ou na enfermaria, após os devidos procedimentos cirúrgicos e clínicos. Assim, na UE, o fisioterapeuta precisa ter foco no essencial, estabelecendo prioridades.

Ficou evidente nos discursos dos fisioterapeutas que os atendimentos na UE seguem essa linha de raciocínio, baseados nas prioridades, o que influenciou nas respostas da entrevista. Desta forma, foi possível identificar quais seriam os conhecimentos e habilidades que os alunos prioritariamente deveriam adquirir para a UE.

Esses conhecimentos e habilidades prioritários, em ordem decrescente de menção, foram: ventilação mecânica (VM), participação na intubação orotraqueal (IOT) e verificação do posicionamento correto do tubo orotraqueal (TOT), manobras de Ressuscitação Cardiopulmonar (RCP) e avaliação dos exames complementares de imagem, principalmente radiografias, e laboratoriais, principalmente a gasometria arterial $(\mathrm{GA})$.

"[...] a meu ver o que ele [o aluno] tem que saber mesmo é atuar na intubação, numa parada cardiorrespiratória e realizar um bom transporte e saber admitir e corrigir a gasometria arterial e ventilação mecânica dos pacientes" (Fisio 1).

"de imediato mesmo é dominar uma intubação orotraqueal, é avaliar o paciente de maneira correta saber se aquele tubo orotraqueal está adequado em posicionamento, ventilação mecânica, raio $X$, exames laboratoriais, então, uma gasometria arterial, porque isso é primordial [...]" (Fisio 9).

Os demais conhecimentos e habilidades, além dos considerados prioritários, foram: manobras e técnicas de Fisioterapia Respiratória; procedimentos de aspiração endotraqueal (tubo orotraqueal ou cânula de traqueostomia); atuação no transporte intra-hospitalar; avaliação do paciente (principalmente a avaliação do estado hemodinâmico e ausculta pulmonar); atuação fisioterapêutica no pré e pós-operatório conforme o perfil de cada serviço de UE.

"É importante ter um conhecimento, assim, é importante a pessoa gostar de fisioterapia respiratória, porque, querendo ou não, na urgência e emergência, a gente vai trabalhar diretamente com isso, com a fisioterapia respiratória" (Fisio 7).

"tem que manobrar, fazer manobras de fisioterapia respiratória, aspiração, mas, fora isso, o básico seria a ventilação mecânica" (Fisio 2).

"tem que saber algo sobre alterações hemodinâmicas, ventilação mecânica, fazer uma análise de exames laboratoriais, ter uma boa noção de diagnóstico por imagem, saber as terapias manuais, terapias de fisioterapia respiratória no caso" (Fisio 5).

Os conhecimentos e habilidades mencionados pelos fisioterapeutas estão no âmbito da fisioterapia hospitalar geral e pode-se inferir que fazem parte do escopo de atuação dos especialistas em Fisioterapia Respiratória, Fisioterapia em Terapia Intensiva e Fisioterapia Cardiovascular, o que vai ao encontro do perfil dos fisioterapeutas entrevistados. 
Foi descrito por Almeida ICN, et al. (2017) o perfil clínico-epidemiológico dos pacientes atendidos pelos fisioterapeutas na UE e demonstrou que $62,3 \%(n=109)$ foram classificados com risco vermelho, os tipos de trauma mais frequentes foram o traumatismo cranioencefálico (TCE) com 65,71\% ( $n=115)$, o trauma ortopédico com $17,71 \%(n=31)$ e as emergências clínicas (não traumáticas) com $14,86 \%(n=26)$. Entre os pacientes vítimas de trauma, os mecanismos mais frequentes foram: acidente de moto $32,57 \%$ ( $n=57$ ), ferimento por arma de fogo $16 \%(n=28)$ e atropelamento $10,86 \%(n=19)$. Em relação às condutas fisioterapêuticas, observou-se: controle de ventilação mecânica em $88 \%$ dos casos $(n=154)$, aspiração endotraqueal em $60,57 \%(n=106)$, montagem do ventilador mecânico em $49,14 \%(n=86)$, transporte intrahospitalar em 42,29\% ( $n=74)$ e técnicas de fisioterapia respiratória em 31,43\% ( $n=55)$.

Assim, os pacientes atendidos pelos fisioterapeutas na UE são de perfil crítico, o que condiz com as condutas realizadas e, consequentemente, com os conhecimentos e habilidades que os alunos precisariam adquirir no estágio supervisionado. Semelhantemente ao observado nesta pesquisa, estudos realizados em outros três hospitais públicos brasileiros, nos estados de São Paulo, Goiás e Minas Gerais, constataram que os fisioterapeutas promoveram mais frequentemente as seguintes condutas, em ordem decrescente de frequência: oxigenoterapia, ventilação mecânica invasiva ou não invasiva, manobras de técnicas de fisioterapia respiratória (OGAWA KYL, et al., 2009; TAQUARY SAS, et al., 2013; GONÇALVES ACS, 2014).

A atuação de fisioterapeutas na UE parece depender do conhecimento e habilidades que perpassam por várias "subdisciplinas" da profissão, porém, a definição do quanto cada disciplina contribui para essa atuação, ou o quanto uma disciplina é "mais importante" que outra, é uma tarefa difícil. É necessário conhecer o perfil específico de cada serviço de UE onde o fisioterapeuta atua. A formação generalista e o emprego de conhecimentos e habilidades de várias disciplinas da Fisioterapia são importantes para a atuação na UE e não está claro na literatura se habilidades específicas favorecem ou não os resultados positivos da atuação fisioterapêutica na UE (LEFMANN S e CRANE J, 2016; KILNER E, 2011).

\section{Atitudes necessárias para atuar na a importância do trabalho em equipe}

Uma competência que precisa ser desenvolvida pelo aluno para atuar na UE, bastante enfatizada pelos fisioterapeutas em vários momentos das entrevistas, foi o trabalho em equipe, apontado como um requisito básico para o desenvolvimento profissional nessa área:

"Em relação à equipe tem que ser uma relação multiprofissional para que haja uma discussão e que essa discussão sobre o estado clínico do paciente e a condução clínica chegue em um objetivo" (Fisio 11).

"tem que saber trabalhar em equipe, ter noção de que a conduta ali é multiprofissional, não vai depender só da fisioterapia" (Fisio 10).

"você trabalhando da maneira, acho que a palavra certa é em conjunto, um ajudando o outro, porque ali a gente precisa realmente bastante do técnico de enfermagem, principalmente, e do enfermeiro também" (Fisio 9).

"ele [o aluno] tem que se comunicar com o enfermeiro, tem que se comunicar com o técnico de enfermagem, até porque em uma urgência e emergência todo mundo precisa de todo mundo" (Fisio 2).

Segundo Salviano AF, et al. (2017), o trabalho em equipe na UE enfatiza a complementaridade entre diferentes profissionais, sem anular a especificidade de cada um, e promove a "compreensão integral do ser humano e do processo saúde-doença". O trabalho em equipe na UE é realizado por diferentes profissionais que trabalham paralelamente na avaliação dos pacientes, elaboração dos planos terapêuticos e condutas assistenciais, com objetivos comuns, o que contribui para a maior resolutividade da alta demanda de pacientes. Profissionais na UE devem trabalhar em conjunto e compartilhar habilidades e conhecimentos, em uma relação de colaboração mútua (MORPHET J, et al., 2016).

Na Suécia, como parte dos cursos de graduação da área da saúde, são realizados programas exitosos de educação interprofissional, nos quais estudantes de diferentes cursos (principalmente Medicina, Enfermagem 
e Fisioterapia), interagem no ambiente de aprendizagem e prestam assistência ao mesmo paciente em conjunto. O objetivo desses programas é que os estudantes aprendam uns com os outros e conheçam a atuação das outras profissões, o que contribui para a melhora da qualidade da assistência e para o trabalho em equipe. Existem programas de educação interprofissional específicos para UE, cujo lema é "Learning together to be able to work together" ("aprender juntos para serem capazes de trabalhar juntos", em uma tradução livre) (ERICSON A, et al., 2017). Os membros da equipe multiprofissional mais citados pelos fisioterapeutas foram o enfermeiro, técnico de enfermagem e médico, o que era esperado, porque esses são os membros da equipe fixa da UE.

Nessa categoria houve destaque para outra competência, diretamente relacionada ao trabalho em equipe, que é a comunicação eficaz:

"Trabalhar em equipe multiprofissional, tanto com o médico principalmente, quanto com a enfermeira, com o técnico de enfermagem, perguntar para poder evoluir o paciente, né? Ele tem que saber dialogar bem com todos da equipe multiprofissional para poder atender da melhor forma o paciente, de forma humanizada" (Fisio 1).

A comunicação é considerada um fator crucial na assistência multiprofissional na UE e as falhas de comunicação podem levar a resultados menos eficazes ou, até mesmo, a resultados negativos para a condição de saúde dos pacientes (ABOURBIH D, et al., 2015; NOGUEIRA JWS e RODRIGUES MCS, 2015). Tanto o trabalho em equipe quanto a comunicação são considerados fatores determinantes para a melhoria da assistência e para garantia da segurança do paciente, o que é fundamental no contexto da UE e foi evidenciado pela experiência dos fisioterapeutas entrevistados.

"O aluno que sai daqui para o serviço tem que ter isso certo: que ninguém é nada sem ninguém. Tem que trabalhar, todo mundo tem que conversar o tempo inteiro com a enfermeira, com o médico..." (Fisio 3).

"eu acho que o mínimo é ter um convívio profissional mínimo ali para não querer fazer as coisas de forma isolada, sozinho, faço e aí não comunico nada para a equipe multiprofissional e aí saio. [...] se não, eu não vou estar contribuindo de fato para a recuperação do paciente" (Fisio 7).

A interação e troca de informações e experiências com os outros profissionais é importante, com feedback mútuo entre os membros da equipe. Isso contribui para a maior resolutividade e para a melhora da qualidade da assistência prestada ao usuário, em uma visão humanizada.

Além disso, um comportamento que demonstre segurança foi valorizado pelos fisioterapeutas, porém, nesta categoria, mais voltada para a interação com a equipe:

"é aquela coisa: nem todo mundo vai gostar de ti, mas, pelo menos, você sendo um bom profissional no que está fazendo, você vai mostrar para todo mundo que você sabe, [...] você vai conseguir tocar um serviço em qualquer lugar que você estiver dentro das tuas competências" (Fisio 8).

$\mathrm{E}$, finalmente, uma atitude de resiliência frente às limitações do serviço, com criatividade e capacidade de improvisação, foi apontada como uma atitude importante na UE:

"A gente, às vezes não têm recurso, então tem que ser um profissional comprometido e que saiba fazer o que ele está fazendo e que saiba lidar com essa falta de recursos, que tenha que improvisar" (Fisio 4);

"Ele tem que ser proativo e tem que usar os materiais que nós temos aqui. Algumas vezes a gente não vai ter disponível material para aquele paciente, mas a gente tem que enquadrar conforme a necessidade" (Fisio 6).

A visão dos fisioterapeutas entrevistados, nesta categoria, foi semelhante ao que Lebec M e Jogodka C (2009) consideram: que a segurança, o trabalho em equipe e a capacidade de comunicação eficaz, além da 
flexibilidade e capacidade de adaptação à natural variação do fluxo de pacientes na UE, são necessários ao fisioterapeuta que trabalha nesse setor. Estas compõem, portanto, o rol de atitudes esperadas do aluno ao final do estágio em UE.

Como limitações da pesquisa, podem ser citados o número reduzido de profissionais $(n=11)$, e a realização da coleta de dados em único centro, pois é possível que resultados encontrados não possam ser expandidos para outros serviços com perfis diferentes de pacientes.

\section{CONCLUSÃO}

A experiência dos fisioterapeutas entrevistados permitiu a identificação de um rol de conhecimentos, habilidades e atitudes que, ao serem articulados e mobilizados pelo aluno, podem contribuir para a resolução dos problemas advindos do cotidiano da UE. Espera-se que essas informações contribuam para o planejamento de estratégias de ensino-aprendizagem voltadas para o campo da Fisioterapia em Urgência e Emergência, na perspectiva de uma formação generalista. Considera-se que outros estudos precisam ser desenvolvidos na área da UE, com objetivo de constante aprimoramento dos processos de formação, no nível de graduação e níveis posteriores, o que certamente contribuirá para a expansão desse campo de atuação do fisioterapeuta e aproximação entre prática e teoria.

\section{REFERÊNCIAS}

1. ABOURBIH D, et al. Communication between nurses and physicians: Strategies to surviving in the emergency department trenches. Emergency Medicine Australasia, 2015; 27: 80-82.

2. ALMEIDA ICN, et al. Atuação da Fisioterapia na Urgência e Emergência de um hospital referência em trauma e queimados de alta e média complexidade. Revista da Universidade Vale do Rio Verde, 2017; 15: 791-805.

3. ALVES AN. A importância da atuação do fisioterapeuta no ambiente hospitalar. Ensaios e Ciência: Ciências Biológicas, Agrárias e da Saúde, 2012; 16(6): 173-184.

4. ERICSON A, et al. Interprofessional education in a student-led emergency department: A realist evaluation. Journal of Interprofessional Care, 2017; 31: 199-206.

5. FLEMING-MCDONNELL D, et al. Physical Therapy in the Emergency Department: Development of a Novel Practice Venue. Physical Therapy, 2010; 90: 420-426.

6. FRAGA GP, et al. A situação do ensino de urgência e emergência nos cursos de graduação de medicina no Brasil e as recomendações para a matriz curricular. In: Projeto ABEM: Diretrizes curriculares. Disponível em: https://bit.ly/2A3JJ5I. Acesso em: 9 nov. 2018.

7. FRUTH SJ, WILEY S. Physician Impressions of Physical Therapist Practice in the Emergency Department: Descriptive, Comparative Analysis Over Time. Physical Therapy, 2016; 96: e20150306.

8. GONÇALVES ACS. Perfil clínico dos pacientes atendidos pelo serviço de fisioterapia na unidade de urgência e emergência de um hospital público de Minas Gerais. ASSOBRAFIR Ciência, 2014; 5(3): 55-62.

9. JESUDASON C, et al. A physiotherapy service to an emergency extended care unit does not decrease admission rates to hospital: a randomised trial. Emerg. Med. J., 2012; 29: 664-669.

10. JIBUIKE O, et al. Management of soft tissue knee injuries in an accident and emergency department: the effect of the introduction of a physiotherapy practitioner. Emerg Med J, 2003; 20: 37-39.

11. KILNER E, SHEPPARD L. The "lone ranger": a descriptive study of physiotherapy practice in Australian emergency departments. Physiotherapy, 2010; 96: e20674658.

12. KILNER E. What evidence is there that a physiotherapy service in the emergency department improves health outcomes? A systematic review. J Health Serv Res Policy, 2011; 16(1): e21186320.

13. LEBEC M, JOGODKA C. The physical therapist as a musculoskeletal specialist in the emergency department. Journal of Orthopaedic and Sports Physical Therapy, 2009; 39: e102519.

14. LEFMANN S, CRANE J. Establishing the diverse value of the emergency department physiotherapist: editorial. Journal of Physiotherapy, 2016; 96: e101016.

15. MARINHO-ARAÚJO CM, ALMEIDA LS. Approach to competences, human development and higher education. Revista Psicologia: Teoria e Pesquisa, 2016, 32: e32212.

16. MORAES EN. Atenção à saúde do Idoso: Aspectos Conceituais. Brasília: Organização Pan-Americana da Saúde, 2012; 98 p.

17. MORAES JC. Formação e competências para classificação de risco em Urgência e Emergência obstétrica. Dissertação (Mestrado em Ensino na Saúde) - Universidade Federal de Goiás, Goiânia, 2013; 137p. 
18. MORPHET J, et al. Using transprofessional care in the emergency department to reduce patient admissions: $\mathrm{A}$ retrospective audit of medical histories. Journal of Interprofessional care, 2016; 30: 226-231.

19. NOGUEIRA JWS, RODRIGUES MCS. Comunicação efetiva no trabalho em equipe em saúde: desafio para a segurança do paciente. Cogitare Enfermagem, 2015; 20: e105380.

20. OGAWA KYL, et al. Intervenção fisioterapêutica nas emergências cardiorrespiratórias. O Mundo da Saúde, 2009; 33: 457-466.

21. PINES J, et al. International Perspectives on Emergency Department Crowding. Academic Emergency Medicine, 2011; 18: 1358-1370.

22. SALT E. Effectiveness of Musculoskeletal Emergency Physiotherapy Practitioners. Open Journal of Therapy and Rehabilitation, 2016; 4(1): e 43013.

23. SALVIANO AF, et al. Atuação interdisciplinar em um serviço hospitalar de urgência e emergência pediátrica. Revista de Ciências da Saúde Nova Esperança, 2017; 15: 27-33.

24. SUTTON M. Primary-contact physiotherapists manage a minor trauma caseload in the emergency department without misdiagnoses or adverse events: an observational study". Journal of Physiotherapy, 2015; 61: 77-80.

25. TAQUARY SAS, et al. Perfil clínico e atuação fisioterapêutica em pacientes atendidos na emergência pediátrica de um hospital público de Goiás. Fisioterapia e Pesquisa, 2013; 20: 262-267.

26. TAYLOR N, et al. Primary contact in emergency departments can reduce length of stay for patients with peripheral musculoskeletal injuries compared with secondary contact physiotherapy: a prospective non-randomised controlled trial. Physiotherapy, 2011; 97: 107-114. 